\title{
Maximal Ratio Combining Performance Analysis in Spatially Correlated Rayleigh Fading Channels with Imperfect Channel Knowledge
}

\author{
D. B. Smith* and T. D. Abhayapala* $\dagger$ \\ ${ }^{*}$ Wireless Signal Processing, National ICT Australia (NICTA) \\ Locked Bag 8001, Canberra ACT 2601, AUSTRALIA \\ (e-mail: David.Smith@nicta.com.au) \\ ${ }^{\dagger}$ Research School of Information Sciences and Engineering \\ the Australian National University, ACT 200, AUSTRALIA \\ (e-mail: Thushara.Abhayapala@anu.edu.au)
}

\begin{abstract}
New Results for the performance analysis of maximal ratio combining (MRC) using BPSK modulation in spatially correlated Rayleigh fading channels with imperfect channel knowledge are presented in terms of antenna array configuration and parameters of scatterer distributions. The utility of recently developed expressions for bit error probability is enhanced by application of a spatial correlation formulation for arbitrarily spaced antennas and general scatterer distributions. The results of performance analysis give valuable insight into the performance of MRC in realistic Rayleigh fading scenarios with imperfect channel state information for both isotropic and non-isotropic fading scenarios.
\end{abstract}

\section{INTRODUCTION}

Maximal ratio combining (MRC) represents a theoretically optimal combiner over fading channels as a diversity scheme in a communication system. Theoretically, multiple copies of the same information signal are combined so as to maximize the instantaneous SNR at the output [1]. It is a very effective technique to mitigate the effects of severe fading in wireless communications.

System designs often assume that the fading is independent across multiple diversity channels of the combiner. Physical constraints often restrain the use of antenna spacing that is required for independent fading across multiple antennas [2]. Therefore it is necessary to consider spatial correlation characteristics between the antennas when implementing MRC.

In addition, system designs often assume that the channel coefficients are known at the receiver, and there are no channel estimation errors; although, in practice the channel coefficients need to be estimated at the receiver. These must be estimated either by the use of known pilot signals or in a blind manner. This can have a deleterious effect on the performance of MRC.

Recently analytical expressions for the bit error probability with BPSK modulation have been derived for typical scenarios where there is correlation with imperfect channel knowledge,

National ICT Australia is funded through the Australian Government's Backing Australia's Ability initiative, in part through the Australian Research Council. This research is partially supported by the Australian Research Council grant DP0343804. accounting for distinct eigenvalues in the correlation matrix [3], and closely spaced or non-distinct eigenvalues [4]. In this paper, for the first time, both the physical characteristics of scatterer distributions and the antenna array inputs to the combiner, are related to the performance of MRC in typical scenarios.

In [5] expressions are given for the spatial correlation of distributions of scatterers which can be applied to various non-isotropic scatterer distributions over multiple antennas. In this contribution, the spatial correlation formulation in [5] is applied with MRC of BPSK modulation to typical antenna configurations for 4 to 6 receive antennas in two typical non-isotropic Rayleigh fading environments, and a Rayleigh isotropic scattering scenario where it is assumed that there is imperfect channel knowledge at the receiver using the results from [3] and [4].

This paper is organized as follows. The next section describes the system model used. The expressions for the bit error probability are described in Section 3. Section 4 describes the spatial correlation formulation at arbitrarily spaced antennas. Section 5 presents analysis of MRC with standard antenna configurations as inputs for different scattering scenarios. The final section provides some concluding remarks.

\section{System MODEL}

The BPSK modulated symbols $a_{n}^{(d)} \in\left\{+\sigma_{d},-\sigma_{d}\right\}$ are transmitted with $\left\|a_{n}^{(d)}\right\|^{2}=\sigma_{d}^{2}$ and $n$ denoting the time index. Then the vector $\boldsymbol{y}_{n}=\left[y_{0, n}, \ldots, y_{L-1, n}\right]^{T}$ comprising the $L$ spatio-temporal diversity branches, which are used for maximal ratio combining (MRC) at the receiver, may be modelled as

$$
\boldsymbol{y}_{n}=a_{n}^{(d)} \boldsymbol{h}+\boldsymbol{v}_{n}
$$

where $\boldsymbol{h}$ denotes the effective vector of channel transmission coefficients, $\boldsymbol{h}=\left[h_{1}, \ldots, h_{L}\right]^{T}$, and $\boldsymbol{v}_{n}$ denotes the noise vector, $\boldsymbol{v}_{n}=\left[v_{1, n}, \ldots, v_{L, n}\right]^{T}$, respectively [4].

Incorporating the assumption of Rayleigh fading, the vector of channel transmission coefficients $\boldsymbol{h}$ is assumed to have a 
zero mean complex Gaussian distribution with covariance $\boldsymbol{R}$, with a distribution $\boldsymbol{h} \sim \mathcal{N}_{C}(\mathbf{0}, \boldsymbol{R})$. The noise is assumed to be additive complex Gaussian noise with distribution $\boldsymbol{v}_{n} \sim$ $\mathcal{N}_{C}\left(\mathbf{0}, \sigma_{v}^{2} \boldsymbol{I}_{L}\right)$, where $\boldsymbol{I}_{L}$ is the identity matrix of size $L$.

We assume that the spatial correlation between two received signals from any two of the combiner inputs from antennas located at positions $\boldsymbol{x}_{k}$ and $\boldsymbol{x}_{\ell}$, following from [5], is

$$
\rho_{k, l}=\frac{E\left\{h_{k} h_{l}^{*}\right\}}{\sqrt{E\left\{h_{k} h_{k}^{*}\right\} E\left\{h_{l} h_{l}^{*}\right\}}} .
$$

where $(\cdot)^{*}$ denotes the conjugate. The spatial correlation $\rho_{k, l}$ make up the elements of $\boldsymbol{R}$, if we assume that $\boldsymbol{R}$ is normalized.

Following from [4] $\boldsymbol{y}_{n}$ also has a zero mean complex Gaussian distribution with covariance matrix

$$
\boldsymbol{K}_{\boldsymbol{y}_{n}}=E\left\{\boldsymbol{y}_{n} \boldsymbol{y}_{n}^{H}\right\}=\sigma_{d}^{2} \boldsymbol{R}+\sigma_{v}^{2} \boldsymbol{I}_{L}
$$

where $(\cdot)^{H}$ represents the conjugate transpose. The noise samples and the channel coefficients are assumed to be independent.

Following from [3] and [4] we assume that the receiver does not have perfect channel state information (CSI) and that a noisy channel estimate $\hat{h}$ is available. The channel estimate is obtained by maximum-likelihood (ML) channel estimation using a block of $M$ pilot symbols $a_{t}^{(p)} \in\left\{+\sigma_{p},-\sigma_{p}\right\}$ with variance $\sigma_{p}^{2}$. This is given in [6];

$$
\hat{\boldsymbol{h}}=\boldsymbol{h}+\frac{1}{M \sigma_{p}^{2}} \sum_{t=0}^{M-1} a_{t}^{(p) *} \boldsymbol{v}_{m}
$$

As in [4] the additive noise in (1) and the channel estimation noise in (4) are assumed to be independent giving the covariance matrix of $\boldsymbol{y}_{n}$ and $\hat{\boldsymbol{h}}$ as

$$
\boldsymbol{K}_{\boldsymbol{y}_{n} \hat{\boldsymbol{h}}}=E\left\{\boldsymbol{y}_{n} \hat{\boldsymbol{h}}^{H}\right\}=\sigma_{d} \boldsymbol{R} .
$$

The MRC decision variable of the diversity branches, $d_{n}$, is given as

$$
d_{n}=\operatorname{Re}\left\{\hat{\boldsymbol{h}}^{H} \boldsymbol{y}_{n}\right\}
$$

\section{BIT ERROR PROBABILITY FOR IMPERFECT CHANNEL KNOWLEDGE}

In this section the bit error probability for BPSK is given from the derivation in [3] and the subsequent derivation in [4].

If we define

$$
\boldsymbol{r}_{n}=\left[\begin{array}{l}
\hat{\boldsymbol{h}}_{n} \\
\boldsymbol{y}_{n}
\end{array}\right], \boldsymbol{A}=\frac{1}{2}\left[\begin{array}{ll}
0 & 1 \\
1 & 0
\end{array}\right] \otimes \boldsymbol{I}_{L},
$$

we obtain the $2 L$ eigenvalues, $\lambda_{l}$, of $\boldsymbol{A} \boldsymbol{K}_{\boldsymbol{r}}$, where $\boldsymbol{K}_{\boldsymbol{r}}=$ $E\left\{\boldsymbol{r}_{n} \boldsymbol{r}_{n}^{H}\right\}$ is the $(2 L \times 2 L)$ covariance matrix of $\boldsymbol{r}_{n}$. The decision variable can be expressed as $d_{n}=\boldsymbol{r}_{n}^{H} \boldsymbol{A} \boldsymbol{r}_{n}$ in hermitian quadratic form. Then, assuming distinct eigenvalues, the bit error probability is obtained from the pdf of $d_{n}$ by integration as

$$
P_{b}=\sum_{\substack{l=1 \\ \lambda_{l}<0}}^{2 L} \prod_{\substack{k=1 \\ k \neq l}}^{2 K} \frac{\lambda_{l}}{\lambda_{l}-\lambda_{k}}
$$

If there are non-distinct eigenvalues, or the eigenvalues are very closely spaced and hence the evaluation of (8) becomes numerically unstable, then the bit error probability (BEP) can be calculated directly from the characteristic function of $d_{n}$, [4], as

$$
P_{b}=\frac{1}{2}-\frac{1}{\pi} \int_{0}^{\infty} \operatorname{Im}\left\{\frac{1}{\omega \prod_{l=0}^{2 L-1}\left(1-j \omega \lambda_{l}\right)}\right\} d \omega .
$$

The eigenvalues, $\lambda_{l}$, in (8) and (9) can be found from the eigenvalue decomposition of

$$
\left[\begin{array}{lr}
\boldsymbol{R} & \boldsymbol{R}+\frac{1}{\gamma_{d}} \boldsymbol{I}_{L} \\
\boldsymbol{R}+\frac{1}{\gamma_{p}} \boldsymbol{I}_{L} & \boldsymbol{R}
\end{array}\right]
$$

where

$$
\gamma_{d}=\frac{\sigma_{d}^{2}}{\sigma_{v}^{2}}, \gamma_{p}=\frac{\sigma_{p}^{2}}{\sigma_{v}^{2}}
$$

representing the average bit signal-to-noise ratio (SNR) and the effective pilot-signal-to-noise ratio respectively.

\section{SPATIAl CORRELATION Formulation AT ARBITRARILY SPACED ANTENNAS}

A generalized spatial correlation, $\rho_{k, l}$, of antenna signals is defined for any two inputs, antennas, of an $L$-branch MRC, assuming that antennas are located in the azimuth plane. Conversely the scattered fields arriving at each MRC input are considered to be located in the azimuth plane only. Thus following from (2) and [5] where the 2-D modal expansion in [7] is

$$
\rho_{k, l}=\sum_{m=-\infty}^{\infty} j^{m} \gamma_{m} J_{m}\left(k\left\|\boldsymbol{x}_{\boldsymbol{k}}-\boldsymbol{x}_{\boldsymbol{l}}\right\|\right) e^{j m \phi_{k l}},
$$

where $J_{m}(\cdot)$ is the $m$ th order Bessel function of the first kind, $k=2 \pi / \lambda, \phi_{k, l}$ is the angle of the vector connecting $\boldsymbol{x}_{\boldsymbol{k}}$ and $\boldsymbol{x}_{\boldsymbol{l}}$, which are points in space, and

$$
\gamma_{m}=\int_{0}^{2 \pi} \mathcal{P}(\phi) e^{-j m \phi} d \phi
$$

where $\mathcal{P}(\phi)$ can be considered to be the normalized average power of a signal received from the direction $\phi$. Note that if $L>2, \phi_{k, l}$ must be considered as non-zero, otherwise it can be considered to be zero.

Each $\rho_{k, l}$ thus formulated, can be used to form the covariance matrix $\boldsymbol{R}$, and then find the corresponding eigenvalues, $\lambda_{l}$, of $\boldsymbol{A} \boldsymbol{K}_{\boldsymbol{r}}$ using (10). These can be used to find the corresponding BEP, $P_{b}$, using (8) or (9) as suitable.

\section{ANALYSIS OF MRC WITH STANDARD ANTENNA CONFIGURATION INPUTS AND IMPERFECT CHANNEL KNOWLEDGE}

In this section we analyze the performance of some standard antenna configurations as inputs to an $L=4$ and $L=6$ branch MRC in isotropic and some non-isotropic scattering scenarios based on the results of the previous sections. Some general performance guidelines are obtained on the basis of the BEP for BPSK modulation with imperfect channel knowledge. 
The standard antenna configurations acting as inputs to the MRC are the uniform circular array (UCA) and the uniform linear array (ULA). It is also possible to analyze other antenna configurations with the formulation in this paper. The BEP is derived for a range of antenna apertures in wavelengths $(\lambda)$ for the UCA, where the aperture is the diameter of the UCA, and a range of antenna separations for the ULA. For the nonisotropic distributions, various angular spreads, $\sigma_{a}$, defined as the square root of the variance of the particular distribution, are considered. The angular spread is related to the non-isotropy parameter of the distribution.

In all analysis we consider Rayleigh fading where the average data bit SNR on each of the $L$ channels is assumed to be the same, such that $\gamma_{d}=5 \mathrm{~dB}, l=1, \ldots, L$. In all analysis the angle of incidence from broadside $\beta$ is $60^{\circ}$. Fig. 1 shows the BEP with BPSK for $L=4$ branch and $L=6$ branch MRC for different scattering scenarios for a UCA with a noisy channel estimate represented by an effective pilot SNR $\gamma_{p}=10 \mathrm{~dB}$.

Even with a reasonable channel estimate represented by $\gamma_{p}$ $=10 \mathrm{~dB}$ there is still a significant effect on the BEP of MRC, illustrated best by the difference between the uncorrelated line and the I.I.D. line with perfect CSI in Fig. 1.

It is also clear that for both non-isotropic scattering scenarios over a range of angular spreads that there is a large variation in the BEP. The BEP for $L=4$ branch MRC shown in Fig. 1(a) is significantly more than an order of magnitude greater for an angular spread $\sigma_{a}=5^{\circ}$ compared with $\sigma_{a}=30^{\circ}$ for both non-isotropic scatterer distributions at a UCA antenna aperture of $1 \lambda$. The trend of degradation from larger $\sigma_{a}$ to smaller $\sigma_{a}$ for Fig. 1(b) $(L=6)$, is even more significant.

It is demonstrated in Fig. 1 for the non-isotropic scattering scenarios, even with a large angular spread, there is degradation in the BEP when compared to that of a uniform isotropic distribution.

An $L=4$ and $L=6$ branch MRC for a ULA with a range of antenna separation (the distance in wavelengths, $\lambda$, between adjacent elements of the ULA) is shown in Fig. 2. Similar trends are displayed when compared with Fig. 1. However there are two noteworthy differences. Firstly for smaller array sizes, Fig. 2 shows a greater variation in BEP between the non-isotropic scattering scenarios and the uniform scattering scenario. Secondly, in Fig. 2 there is a uniform improvement in the BEP when there is a uniform isotropic distribution for the ULA when compared with the UCA.

The results for the variation of BEP with angular spread in Fig. 1 and Fig. 2 for non-isotropic scatterer distributions may be directly attributable to a decrease in the spatial correlation. In [8] a decrease in spatial correlation is shown as antenna spacing and/or angular spread increases for nonisotropic distributions. The small variation of the BEP for the same angular spread is also explained in [8] by the distribution variance dominating correlation, and not the choice of nonisotropic distribution.

In Fig. 3 and Fig. 4 the effect of various $\gamma_{p}$, i.e. levels of

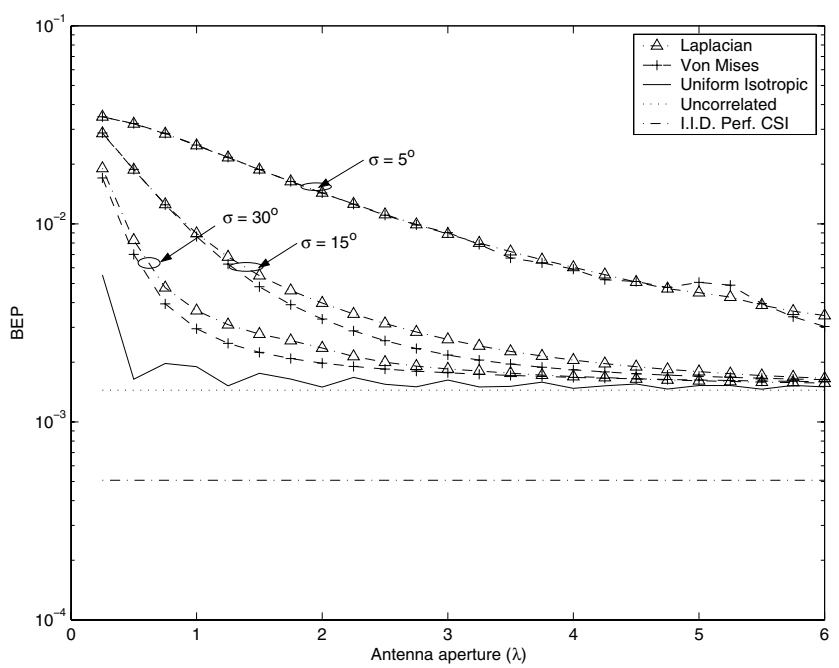

(a) $L=4$ branch MRC

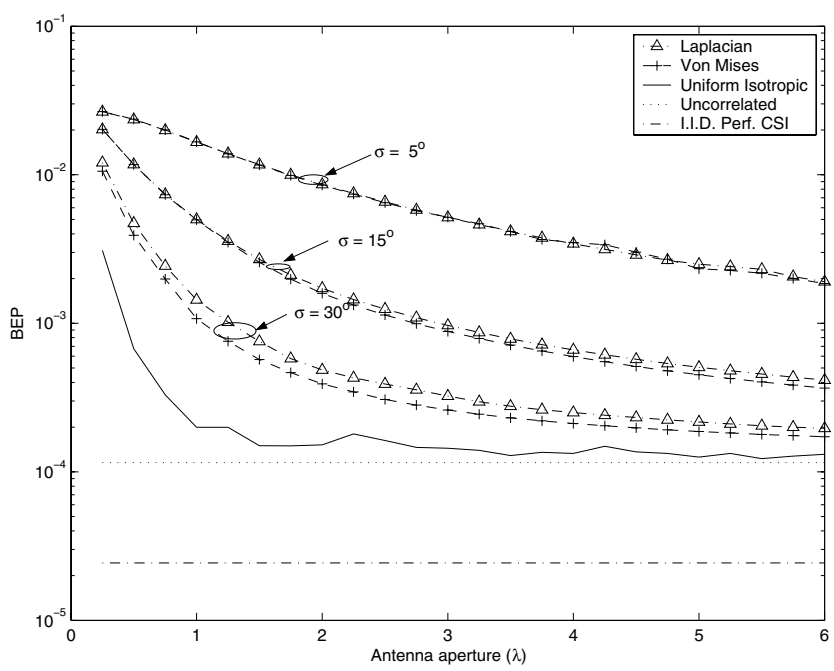

(b) $L=6$ branch MRC

Fig. 1. BEP with MRC for BPSK modulation for Laplacian and Von Mises distributions of various $\sigma_{a}, \beta=60^{\circ}$, and a uniform isotropic distribution, $\gamma_{d}=5 \mathrm{~dB}$ and $\gamma_{p}=10 \mathrm{~dB}$. Inputs to the MRC are from a UCA. MRC for uncorrelated channels, and also $L$ I.I.D. channels with perfect CSI are shown for reference

noisiness in the channel estimate, is shown for a Laplacian scatterer distribution (which is non-isotropic) with an angular spread, $\sigma_{a}=15^{\circ}$ for $L=4$ branch MRC, $\gamma_{d}=10 \mathrm{~dB}$, with a UCA as input in Fig. 3, and a ULA as input in Fig. 4. The case for $\gamma_{p}=\infty$, perfect CSI, or a noiseless estimate, is also shown. In both cases there is a clear, marked, degradation as the estimate based on the pilot signal becomes noisier. It is also clear that the trend of improvement related to increasing antenna aperture is similar for all values of $\gamma_{p}$ with the same distribution.

Thus, with respect to the scenarios outlined in this section the performance of MRC is optimised for larger antenna apertures (or larger adjacent antenna separation). There is little difference between using a ULA and a UCA as the 


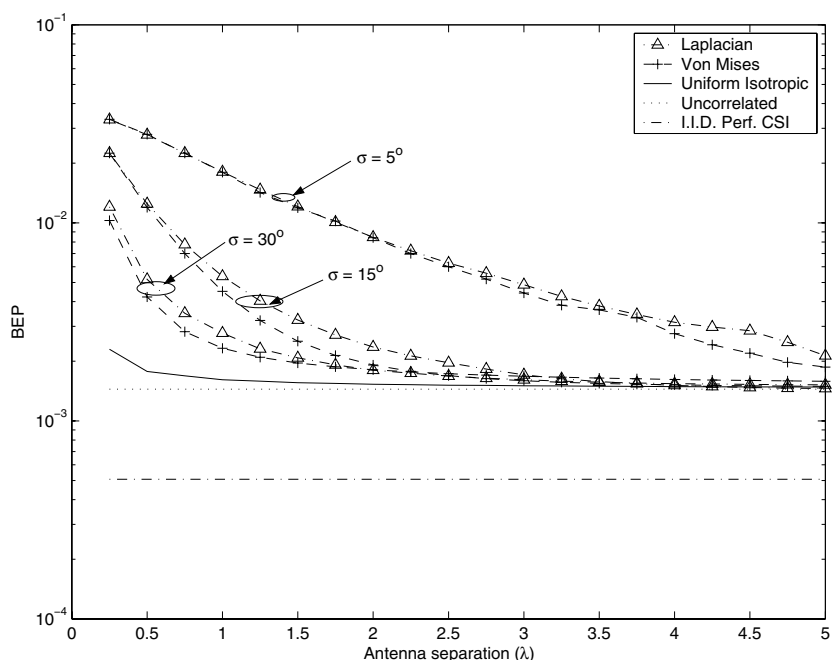

(a) $L=4$ branch MRC

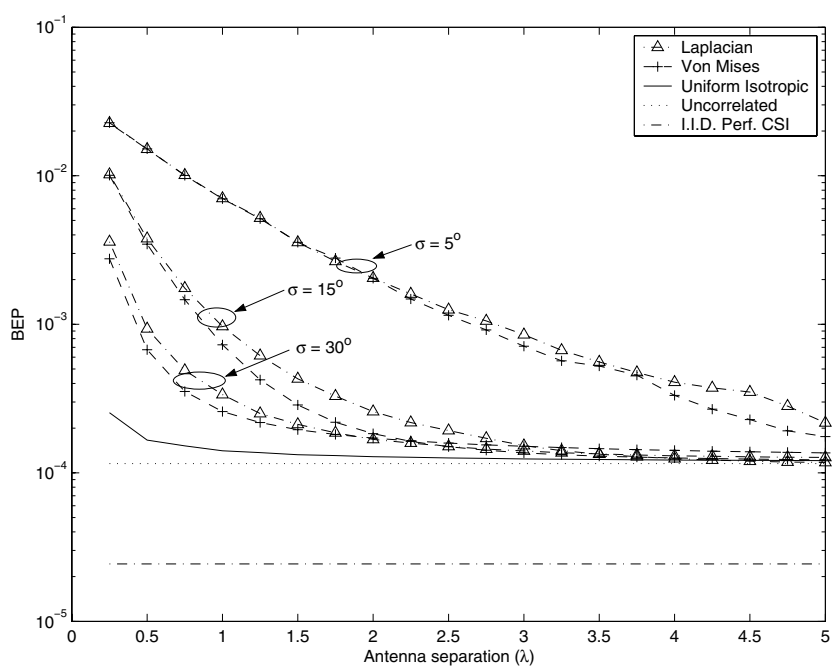

(b) $L=6$ branch MRC

Fig. 2. BEP with MRC of BPSK modulation for Laplacian and Von Mises distributions of various $\sigma_{a}, \beta=60^{\circ}$, and an isotropic distribution, $\gamma_{d}=5 \mathrm{~dB}$ and $\gamma_{p}=10 \mathrm{~dB}$. Inputs to the MRC are from a ULA. MRC for uncorrelated channels, and also 4 I.I.D. channels with perfect CSI are shown for reference

antenna configuration at the input, although for smaller array sizes the ULA may be the preferable array. Furthermore, the similar distribution variance of the Von Mises and Laplacian distributions, the non-isotropic distributions analyzed herein, is demonstrated as dominating the MRC performance, therefore the performance shows very similar characteristics for these different distributions.

\section{Concluding Remarks}

The results in this paper give useful insight into aspects of practical implementation of MRC in Rayleigh fading channels with typical antenna configurations and BPSK modulation. Understanding of the effects of non-isotropy, antenna shape and size may lead to better implementations of receivers for MRC in Rayleigh fading channels. Further investigation into

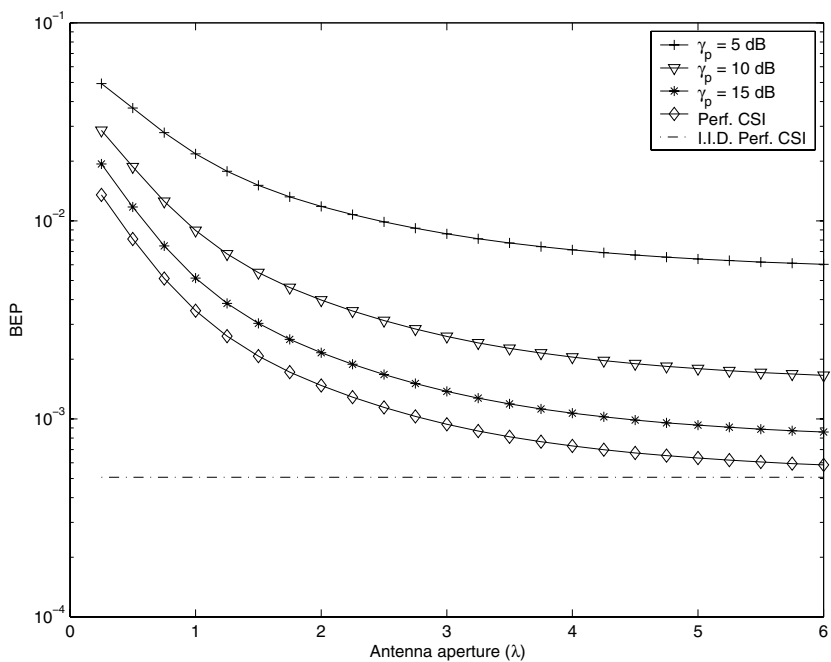

Fig. 3. BEP with 4 branch MRC of BPSK modulation for Laplacian distribution, $\sigma_{a}=15^{\circ}, \beta=60^{\circ}, \gamma_{d}=5 \mathrm{~dB}$, various $\gamma_{p}$. Inputs to the MRC are from a UCA. MRC for 4 I.I.D. channels with perfect CSI is shown for reference

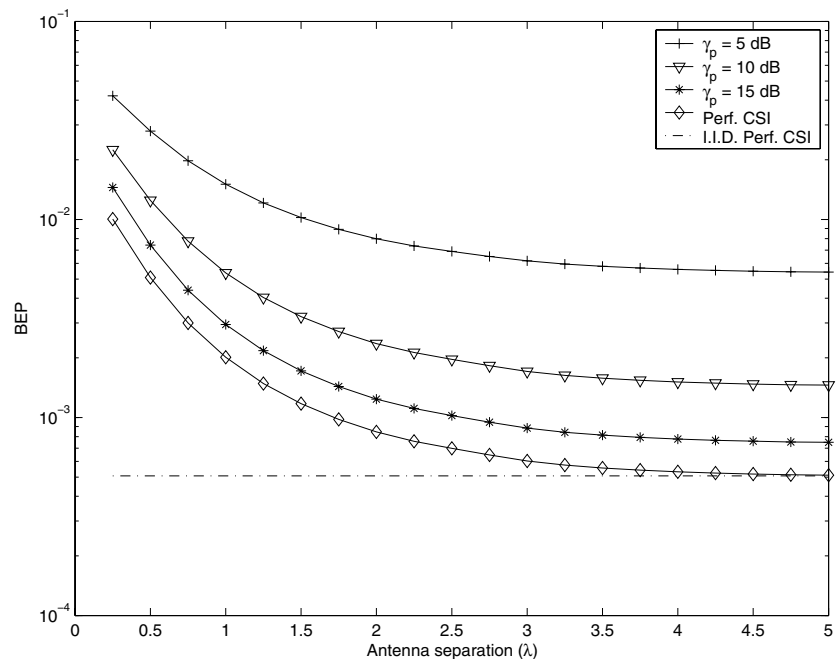

Fig. 4. BEP with 4 branch MRC of BPSK modulation for Laplacian distribution, $\sigma_{a}=15^{\circ}, \beta=60^{\circ}, \gamma_{d}=5 \mathrm{~dB}$, various $\gamma_{p}$. Inputs to the MRC are from a ULA. MRC for $L$ I.I.D. channels with perfect CSI is shown for reference

the performance of other modulation schemes with practical implementations may give extra insight into the general performance of MRC in Rayleigh fading channels.

\section{REFERENCES}

[1] D. Brennan, "Linear diversity combining techniques," Proc. IRE, vol. 47, no. 1, pp. 1075-1102, June 1959.

[2] W.C. Jakes, Microwave Mobile Communications, John Wiley, New York, 1974.

[3] F. A. Dietrich and W. Utschick, "Maximum ratio combining of correlated rayleigh fading channels with imperfect channel knowledge," IEEE Commun. Lett., vol. 7, no. 9, pp. 419-421, September 2003.

[4] L. Schmitt, T. Grundler, C. Schreyoegg, I. Viering, and H. Meyer, "Maximum ratio combining of correlated diversity branches with imperfect channel state information and colored noise," in Proceedings of the 2004 IEEE International Symposium on Spread Spectrum Techniques and Applications, ISSSTA 2004, Sydney, Australia, August 2004, pp. 859-863. 
[5] P. D. Teal, T. D. Abhayapala, and R. A. Kennedy, "Spatial correlation for general distributions of scatterers," IEEE Sig. Proc. Lett., vol. 9, no. 10, pp. 305-308, October 2002.

[6] H. V. Poor, An Introduction to Signal Detection and Estimation, SpringerVerlag, 2 edition, 1994.

[7] D. Colton and R. Kress, Inverse Acoustic and Electromagnetic Scattering Theory, vol. 93, Springer, New York, 2 edition, 1998.

[8] T. S. Pollock, T. D. Abhayapala, and R. A. Kennedy, "Introducing space into MIMO capacity calculations," Journal on Telecommunication Systems, vol. 24 , no. 2-4, pp. 415-436, 2003. 